\title{
TEMPERATURE-DEPENDENT FATIGUE CHARACTERISTICS OF P91 STEEL
}

\author{
Władysław EGNER*, Piotr SULICH**, Stanisław MROZIŃSK'**, Halina EGNER \\ *Institute of Applied Mechanics, Faculty of Mechanical Engineering, Cracow University of Technology, \\ 31-864 Kraków Al. Jana Pawła II 37, Poland \\ "Huta Stalowa Wola S.A., ul. gen. Tadeusza Kasprzyckiego 8, 37-450 Stalowa Wola, Poland \\ ${ }^{* *}$ UTP University of Science and Technology, Faculty of Mechanical Engineering, 85-225 Bydgoszcz ul. Kordeckiego 20, Poland \\ wladyslaw.egner@pk.edu.pl, p.sulich@hsw.pl, stmpkm@utp.edu.pl, halina.egner@pk.edu.pl
}

received 17June 2019, revised 12 May 2020, accepted 15 May 2020

\begin{abstract}
In this paper, the experimental investigations, constitutive description and numerical modelling of low-cycle fatigue behaviour of P91 steel in non-isothermal conditions are presented. First, experimental tests are performed to recognise different aspects of material behaviour. Then, an appropriate constitutive model is developed within the framework of thermodynamics of irreversible processes with internal state variables. The model describes two phases of cyclic softening, related to plastic mechanisms. An important goal of the presented research is to include thermomechanical coupling in the constitutive modelling. Next, the model parameters are identified based on the available experimental data. Some parametric studies are presented. Finally, numerical simulations are performed, which indicate the significant influence of thermomechanical coupling on the response of the constitutive model in thermomechanical fatigue conditions.
\end{abstract}

Key words: low-cycle fatigue, thermomechanical coupling, constitutive modelling

\section{INTRODUCTION}

The interest in prediction of cyclic behaviour of materials and structures is growing in the industry. The $9 \%-12 \% \mathrm{Cr}$ steels are being extensively used as structural materials for the components of reactors and power plants (Fournier et al., 2010). Research on this new steel grade dedicated for operation at elevated temperatures began in the USA at the end of the 1970s. As a result of the extensive research, the optimal chemical composition of steel was determined, which resulted in a good combination of mechanical and thermal properties (Kannan et al., 2013) such as high creep resistance and good ductility, high resistance to thermal fatigue, good weldability and the ability to easily make connections with other ferritic steel grades, good thermal conductivity and low thermal expansion coefficient, and good corrosion resistance and fracture toughness in water-steam and gas environments (in the presence of hydrogen) (Fournier et al., 2008; Nagesha et al., 2002; Sauzay et al., 2005; Kyaw et al., 2016)). P91 steel, which is one of the $9 \%-12 \% \mathrm{Cr}$ steel family members, is perfectly suited for thick-walled pipes or forgings for the construction of boilers with extremely high operating requirements and steam generators, nuclear reactors and other responsible devices operating at temperatures up to $650^{\circ} \mathrm{C}$ (Duda, 2015). P91 steel has been also used for many years in power plant installations during renovations to replace austenitic steel and $21 / 4 \mathrm{Cr}-1$ Mo steel pipes. The benefits of its use are the reduction in the diameter and thickness of tubular elements, and thus their mass, which is the result of the higher creep strength of P91 steel.

During the process of frequent starting up and shutting down of the power plant units, high-temperature components are sub- jected to alternating cyclic changes in temperature and mechanical load, i.e. to thermomechanical fatigue. High-chromium steels subjected to high-temperature loads are subject to the evolution of the microstructure. This has been well documented in Nagesha et al. (2002) and Farragher (2014). This evolution manifests itself in the form of subgrain coarsening, where the boundaries between the martensitic laths and subgrain boundaries disappear (Sauzay et al., 2005), which leads to a coarser microstructure. The subgrain coarsening phenomenon depends on the applied plastic strain (Kyaw et al., 2016). There is also a decrease in dislocation density (Nagesha et al., 2002). As a consequence of these microstructural rearrangements, the $9 \%-12 \% \mathrm{Cr}$ steels commonly exhibit cyclic softening (Kyaw et al., 2016; Farragher, 2014; Shankar et al., 2006; Sulich et al., 2017). This softening is observed regardless of the test temperature and the level of deformation.

The present investigation aims at a qualitative and quantitative description of the cyclic softening of P91 steel during low cycle fatigue at elevated and changing temperatures. Due to the variability in temperature, an important goal of the presented research is to include in the constitutive modelling the thermomechanical coupling. The effect of temperature (and its changes) on the phenomenon of cyclic softening of P91 steel is investigated experimentally, and an appropriate constitutive model is developed. The model parameters are determined here from strain-controlled, low-cycle fatigue tests at several isothermal test temperatures with the use of optimisation software. The results obtained at discrete temperatures are then interpolated into the temperature functions of material characteristics. Then the qualitative and quantitative impact of thermomechanical coupling on the response of the model is illustrated. 


\section{MATERIAL BEHAVIOUR}

Experimental tests were performed on P91 steel specimens cut out of a boiler pipe (see Fig. 1; cf. Sulich et al., 2017). The chemical composition of steel was $0.127 \mathrm{C}, 0.442 \mathrm{Si}, 0.489 \mathrm{Mn}$, $0.017 \mathrm{P}, 0.005 \mathrm{~S}, 8,82 \mathrm{Cr}, 0.971 \mathrm{Mo}, 0.307 \mathrm{Ni}, 0.012 \mathrm{Al}, 0.017$ Co, $0.036 \mathrm{Cu}, 0.074 \mathrm{Nb}, 0.004 \mathrm{Ti}$ and $0.201 \mathrm{~V}$. Low-cycle fatigue tests were strain controlled, with a constant total strain amplitude $\varepsilon_{\text {ac }}$ and a constant temperature $\theta$ in each test. Five levels of total strain amplitude $(0.25 \%, 0.30 \%, 0.35 \%, 0.50 \%$ and $0.60 \%)$ and three levels of temperature $\left(20^{\circ} \mathrm{C}, 400^{\circ} \mathrm{C}\right.$ and $\left.600^{\circ} \mathrm{C}\right)$ were applied. Experiments were performed on the testing machine Instron 8502 equipped with a heating chamber.

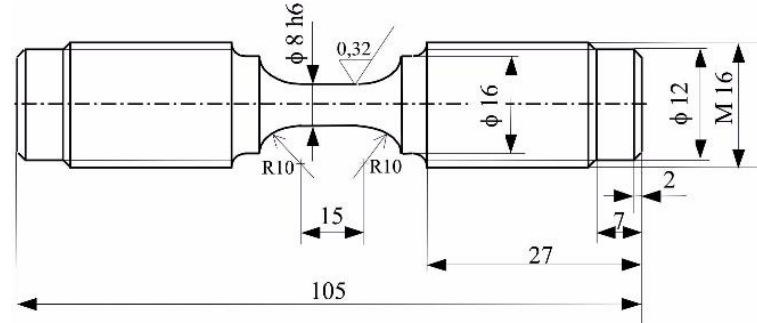

Fig. 1. Shape and dimensions of the tested specimen

a)

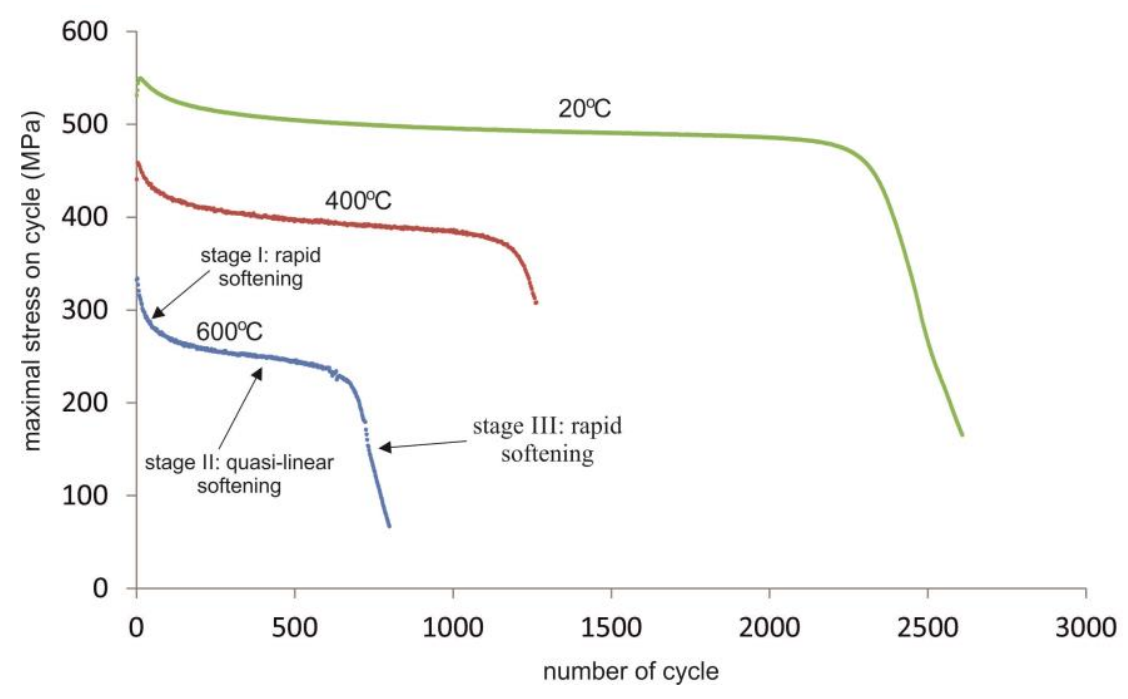

b)

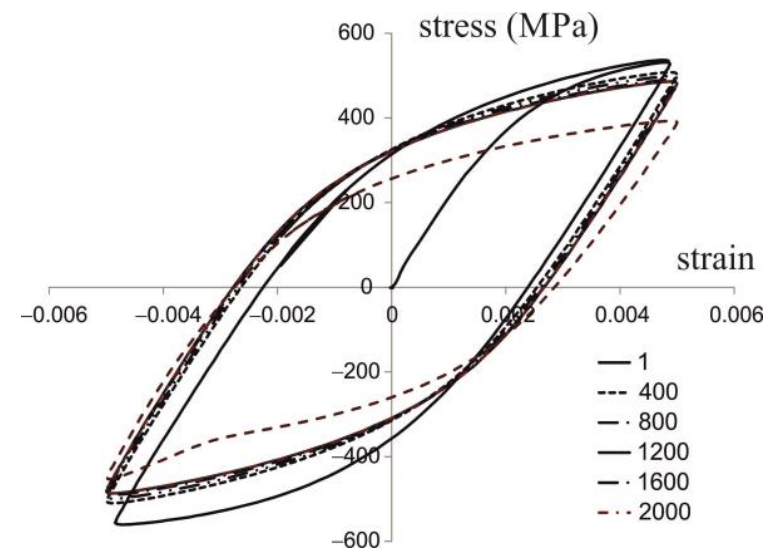

c)

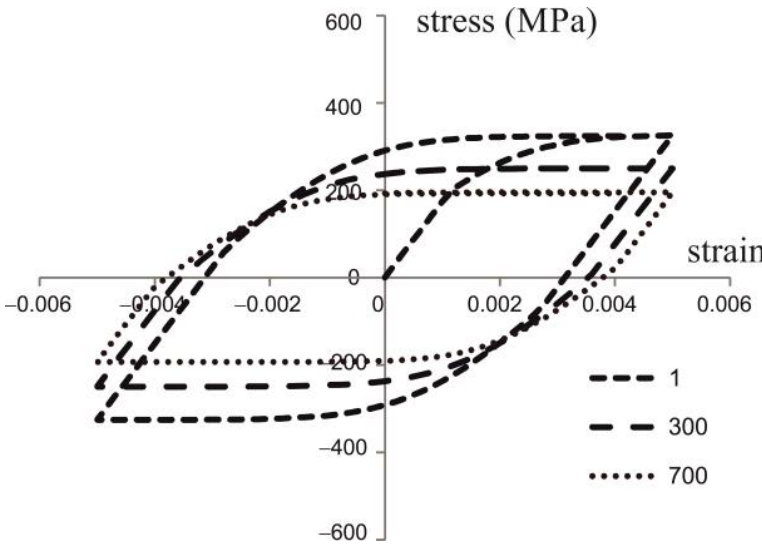

Fig. 2. (a) Maximum stress on cycle versus number of cycles for strain amplitude $\varepsilon_{\mathrm{ac}}=0.5 \%$ and three test temperatures, $20^{\circ} \mathrm{C}, 400^{\circ} \mathrm{C}$ and $600^{\circ} \mathrm{C}$; (b) chosen hysteresis loops for the test temperature $20^{\circ} \mathrm{C}$; (c) chosen hysteresis loops for the test temperature $600^{\circ} \mathrm{C}$

The results (see Fig. 2) indicate that except the first several cycles, the tested steel exhibits cyclic softening, regardless of the testing temperature and strain amplitude (Saad et al., 2011; Li et al., 2016; Mroziński and Golański, 2014; Zhao et al., 2017). This softening could be divided into three phases, which are: the rapid softening phase, followed by the slow quasi-linear softening and finally again fast softening caused by micro-damage development in the material that ultimately leads to failure of the tested sample (Mroziński, 2011). As mentioned before, the tested material generally exhibits an initial slight hardening during the very first cycles, the extent of it being dependent on the test temperature (decreasing with increasing temperature, see Fig. 2a, cf. also
Nagesha et al., 2002; Kruml and Polák, 2001. It may be the effect of several mechanisms, such as the interaction between dislocations and solute atoms, mutual interaction among dislocations and formation of fine precipitates on dislocations during testing. After this very short consolidation phase, the maximum stress on the cycle continuously drops without a saturation period within the range of considered strain amplitudes. For higher plastic strain amplitudes, the tendency to saturation may be observed.

The existing literature suggests various mechanisms responsible for the cyclic softening/hardening of the high-strength steel group. In general, this phenomenon is explained by the modification of dislocation structure and density, carbide morphology, 
density and chemical composition (Zhang et al., 2002). In the study of Jones and Van Den Avyle (1980), it was found for $2.25 \mathrm{Cr}-1 \mathrm{Mo}$ steel that cyclic loading-induced dislocation shuttling accelerates the formation of Mo-C-Mo clusters, leading finally to the replacement of Mo-C pairs, which results in a continuous drop of the stress amplitude. Other mechanisms that have been proposed to explain the phenomenon of cyclic softening of this type of steel include stress reduction associated with the surface oxide film (Kim and Weertman, 1988), and change from the original lath structure to cells or equiaxed subgrains.

There are several parameters that influence the softening amplitude and kinetics (temperature, total or plastic strain amplitude, testing frequency, initial hardness, etc.). The present paper is focused on the description of the influence of variable temperature on the cyclic behaviour in non-isothermal conditions, while the dependence on other test conditions (Xie et al., 2018) and strain range memorisation effect (Zhou et al., 2018) are disregarded.

\section{CONSTITUTIVE MODEL}

\subsection{Basic assumptions}

Cyclic constitutive models have been widely developed for metallic materials and successfully used in academic research and industrial applications during the past decades. In constitutive modelling, the formalism of thermodynamics of irreversible processes with internal state variables and the local state method are often adopted (Skrzypek and Kuna-Ciskał, 2003; Egner and Ryś, 2017; Zhou et al., 2018; Xie et al., 2018). The current state of a material is determined by certain values of some independent variables called variables of state (observable or internal). The choice of internal variables depends on dissipative phenomena taking place in the material that need to be regarded in the theoretical model. The present model is based on the following assumptions: small strains, rate-independent plasticity, mixed isotropic/kinematic plastic hardening.

\subsection{State variables}

For elastic-plastic material exhibiting mixed hardening, the following set of state variables is defined:

$\left\{V_{\alpha}\right\}=\left\{\varepsilon_{i j}^{\mathrm{e}}, \theta ; \alpha_{i j}^{(\mathrm{m})}, r^{(\mathrm{n})}\right\}$

where $\varepsilon_{i j}^{\mathrm{e}}$ are components of the reversible (elastic) strain tensor, $\theta$ is the absolute temperature in Kelvin degrees, $\alpha_{i j}^{(\mathrm{m})}$ correspond to kinematic plastic hardening, while $r^{(\mathrm{n})}$ are related to isotropic plastic hardening. Indexes $\mathrm{m}$ and $\mathrm{n}$ denote the number of different physical mechanisms (different dislocation populations) resulting in the occurrence and nature of hardening (Cailletaud and Saï, 1995; Egner and Egner, 2016). To reduce the number of model parameters, in the present analysis, the simplest case, $\mathrm{m}=\mathrm{n}=$ 1 , is considered.

\subsection{Cyclic softening}

Within the framework of hardening models, isotropic hardening is generally used to express the cyclic evolution of the materi- al's mechanical strength with respect to the plastic flow (Chaboche, 2008). The dimension of the elasticity domain can be controlled with a law of the type:

$R=Q(\theta)\left(1-e^{-b(\theta) r}\right)$

where $b(\theta)$ and $Q(\theta)$ are two coefficients that are material and temperature dependent. However, such a description leads to a typical saturation; therefore, it is not suitable for steels that soften continuously without a saturation period. To take into account the non-saturating cyclic softening observed experimentally in the case of P91 steel, the drag stress $\mathrm{R}$ can be divided into two parts, $R_{1}$ and $R_{2}$ (Zhang et al., 2002; Taleb and Cailletaud, 2010; Egner and Egner, 2014):

$R_{1}=Q(\theta)\left(1-e^{-b(\theta) r}\right), R_{2}=H^{\mathrm{R}}(\theta) r$

The first part, $R_{1}$, corresponds to the strong softening typically taking place during the first hundred of cycles, while the second one, $R_{2}$, allows reflecting continuous softening. Due to the quasilinear character of the second stage of softening, drag stress $R_{2}$ was here adopted in a linear form (Saad et al., 2013, Lu et al., 2015), where $H^{\mathrm{R}}$ reflects the slope of the second stage of cyclic softening (see Fig. 2a).

\subsection{State equations}

The thermodynamic forces conjugated to state variables (1) result from the assumed form of the state potential, which is here the Helmholtz free energy $\rho \psi$, decomposed into thermoelastic and thermoplastic parts (Sulich et al., 2017). In the present analysis, the following state equations define the thermodynamic forces:

$\sigma_{i j}=\rho \frac{\partial \psi}{\partial \varepsilon_{i j}^{\mathrm{e}}}=E_{i j k l}(\theta)\left(\varepsilon_{k l}-\varepsilon_{k l}^{\mathrm{p}}\right)-\beta_{i j}(\theta)\left(\theta-\theta_{0}\right)$

$X_{i j}=\rho \frac{\partial \psi}{\partial \alpha_{i j}}=\frac{2}{3} C(\theta) \alpha_{i j}$

$R=\rho \frac{\partial \psi}{\partial r}=Q(\theta)\left(1-\mathrm{e}^{-b(\theta) r}\right)+H^{\mathrm{R}}(\theta) r=R_{1}+R_{2}$

Symbol $\sigma_{i j}$ denotes the Cauchy stress tensor (thermodynamic force conjugated to elastic strain), $X_{i j}$ denotes back stress (conjugated to plastic kinematic hardening variable $\alpha_{i j}$ ) and drag stress $R$ is conjugated to plastic isotropic hardening variable $r$. Additionally, $E_{i j k l}(\theta)$ denote the components of the elastic stiffness tensor, while $C(\theta), Q(\theta), b(\theta)$ and $H^{R}(\theta)$ stand for temperature-dependent material characteristics.

\subsection{Dissipation potential and evolution equations}

To establish the rate laws, the potential approach is applied here, based on the assumption of the existence of dissipation potential $F$, being closed, convex and scalar-valued function of the thermodynamic forces (4)-(6) and some other possible variables.

To allow for a nonlinear plastic hardening description (Frederick and Armstrong, 2007), the potential of plastic dissipation $F$ is assumed to be not equal to plastic yield surface here (nonassociated plasticity):

$F\left(J_{\alpha}, Z_{\beta}, \theta\right)=f\left(J_{\alpha}, Z_{\beta}, \theta\right)+\frac{3 \gamma(\theta)}{4 C(\theta)} X_{i j} X_{i j}$ 
$f\left(J_{\alpha}, Z_{\beta}, \theta\right)=\sqrt{\frac{3}{2}\left(s_{i j}-X_{i j}\right)\left(s_{i j}-X_{i j}\right)}-\sigma_{\mathrm{y}}(\theta)-R$

where $f\left(J_{\alpha}, Z_{\beta}, \theta\right)$ is the von Mises-type plastic yield function of thermodynamic conjugated forces $J_{\alpha}$ (deviatoric stress $s_{i j}$, back stress $X_{i j}$ and drag stress $R$ ) and material characteristics $Z_{\beta}$ (yield stress $\sigma_{\mathrm{y}}(\theta)$ and material parameters $C(\theta)$ and $\gamma(\theta)$ ). According to the generalised normality rule (Chaboche, 2008), the following rate equations are obtained:

$\dot{\varepsilon}_{i j}^{\mathrm{p}}=\frac{3}{2} \dot{\lambda} \mathrm{p} \frac{s_{i j}-X_{i j}}{\sqrt{\frac{3}{2}\left(s_{k l}-X_{k l}\right)\left(s_{k l}-X_{k l}\right)}}$

$\dot{\alpha}_{i j}=\dot{\varepsilon}_{i j}^{\mathrm{p}}-\frac{3 \gamma}{2 C} X_{i j} \dot{r}$

$\dot{r}=\dot{\lambda}^{p}$

\section{THERMOMECHANICAL COUPLING}

\subsection{Rates of thermodynamic forces}

The constitutive model involves a number of temperaturedependent model characteristics. According to Eqs (4)-(6), the rates of thermodynamic conjugate forces involve explicitly the rate of temperature and are expressed as:

$\dot{\sigma}_{i j}=E_{i j k l}(\theta)\left(\dot{\varepsilon}_{k l}-\dot{\varepsilon}_{k l}^{\mathrm{p}}\right)-$

$\underbrace{\left[-\frac{\partial E_{i j k l}}{\partial \theta}\left(\varepsilon_{k l}-\varepsilon_{k l}^{\mathrm{p}}\right)+\frac{\partial \beta_{i j}}{\partial \theta}\left(\theta-\theta_{0}\right)+\beta_{i j}\right]}_{\text {coupling term } P_{i j}^{\theta}} \dot{\theta}$

$\dot{X}_{i j}=\frac{2}{3} C \dot{\alpha}_{i}+\underbrace{\frac{2}{3} \frac{\mathrm{d} C}{\mathrm{~d} \theta} \alpha_{i j}}_{\text {coupling term } C_{i j}^{\theta}} \dot{\theta}$

$\dot{R}=\left(Q b \mathrm{e}^{-b r}+H^{\mathrm{R}}\right) \dot{r}+$

$+\underbrace{\left[\frac{\mathrm{d} Q}{\mathrm{~d} \theta}\left(1-\mathrm{e}^{-b r}\right)+\left(Q \frac{\mathrm{d} b}{\mathrm{~d} \theta} \mathrm{e}^{-b r}+\frac{\mathrm{d} H^{\mathrm{R}}}{\mathrm{d} \theta}\right) r\right]}_{\text {coupling term } Q^{\theta}} \dot{\theta}$

In the presence of thermoplastic coupling, the rate of a considered thermodynamic force depends, therefore, not only on the rate of internal variable conjugated to it, but also on the rate of temperature. In the following sections, the significance of coupling terms appearing in the kinetic equations (12)-(14)

$P_{i j}^{\theta}=-\frac{\partial E_{i j k l}}{\partial \theta}\left(\varepsilon_{k l}-\varepsilon_{k l}^{\mathrm{p}}\right)+\frac{\partial \beta_{i j}}{\partial \theta}\left(\theta-\theta_{0}\right)+\beta_{i j}$

$C_{i j}^{\theta}=\frac{2}{3} \frac{\mathrm{d} C}{\mathrm{~d} \theta} \alpha_{i j}$

$Q^{\theta}=\frac{\mathrm{d} Q}{\mathrm{~d} \theta}\left(1-\mathrm{e}^{-b r}\right)+\left(Q \frac{\mathrm{d} b}{\mathrm{~d} \theta} \mathrm{e}^{-b r}+\frac{\mathrm{d} H^{\mathrm{R}}}{\mathrm{d} \theta}\right) r$

will be investigated for the case of thermomechanical cyclic loading.

\subsection{Heat balance equation}

To determine the temperature distribution within the body, the heat balance equation is used, which is derived from the first law of thermodynamics by substituting into it the internal energy density together with Fourier's law. The heat balance equation takes the following form (Egner and Egner, 2016) $\left(c_{\varepsilon}^{\theta}\right.$ is the specific heat capacity at a constant strain, $q_{i}$ stands for the heat flux and $r^{\theta}$ is the distributed heat source per unit volume):

$\rho c_{\varepsilon}^{\theta} \dot{\theta}=$

$-q_{i, i}+r^{\theta}+\underbrace{\sigma_{i j} \dot{\varepsilon}_{i j}^{\mathrm{p}}-R \dot{r}-X_{i j} \dot{\alpha}_{i j}}_{\text {mechanical dissipation }}+$

$\theta \underbrace{\left[\frac{\partial R}{\partial \theta} \dot{r}+\frac{\partial X_{i j}}{\partial \theta} \dot{\alpha}_{i j}-P_{i j}^{\theta}\left(\dot{\varepsilon}_{i j}-\dot{\varepsilon}_{i j}^{\mathrm{p}}\right)\right]}_{\text {thermo-mechanical coupling term }}$

According to Eq. (18), determination of the temperature distribution within the body is coupled not only to the total strain rate $\dot{\varepsilon}_{i j}$, but also to the inelastic rates, $\dot{\varepsilon}_{i j}^{\mathrm{p}}$, and fluxes $\dot{\alpha}_{i j}$ and $\dot{r}$.

\subsection{Loading/unloading conditions}

Since the thermodynamic conjugate forces are functions of state variables, the consistency relation for the development of dissipative phenomena takes the following form:

$\dot{f}=\frac{\partial f}{\partial \sigma_{i j}} \dot{\sigma}_{i j}+\frac{\partial f}{\partial X_{i j}} \dot{X}_{i j}+\frac{\partial f}{\partial R} \dot{R}+\frac{\partial f}{\partial \theta} \dot{\theta}=0$

where the term $\frac{\partial f}{\partial \theta}$ explicitly includes the dependence of the initial yield stress $\sigma_{\mathrm{y}}$ on temperature.

Using the chain rule and Eqs (9)-(11) and (12)-(14), the consistency condition becomes:

$\dot{f}=\frac{\partial f}{\partial \sigma_{i j}} \dot{\sigma}_{i j}-\dot{\lambda}^{\mathrm{p}} H-\dot{\theta} S=0$

In the above equation, $H$ is a generalised hardening modulus,

$H=\frac{2}{3} \frac{\partial f}{\partial \sigma_{i j}}\left(C \frac{\partial f}{\partial \sigma_{i j}}-\frac{3}{2} \gamma X_{i j}\right)-\frac{\partial f}{\partial R}\left(Q b \mathrm{e}^{-b r}+H^{\mathrm{R}}\right)$

while $S$ reflects the sensitivity of the yield surface on temperature changes (Egner, 2012):

$S\left\{\begin{array}{l}>0 \Rightarrow \text { yield surf. contracts with increasing } \theta \\ =0 \Rightarrow \text { yield surf. remains constant with increasing } \theta \\ <0 \Rightarrow \text { yield surf. expands with increasing } \theta\end{array}\right.$

where

$S=\frac{\partial f}{\partial \sigma_{i j}} C_{i j}^{\theta}-\frac{\partial f}{\partial R} Q^{\theta}-\frac{\partial f}{\partial \theta}$

Concerning the majority of experiments, the physical meaning has the case when the yield surface contracts with increasing temperature.

Expression (20) determines the consistency multiplier:

$\dot{\lambda}^{\mathrm{p}}(\theta)=\frac{1}{w}[\frac{\partial f}{\partial \sigma_{i j}} E_{i j k l} \dot{\varepsilon}_{k l}-\underbrace{\left(\frac{\partial f}{\partial \sigma_{i j}} P_{i j}^{\theta}+S\right)}_{\text {coupling term }} \dot{\theta}]$

where $w=\frac{\partial f}{\partial \sigma_{i j}} E_{i j k l} \frac{\partial f}{\partial \sigma_{k l}}+H>0$.

Temperature influences the mechanical properties of conventional engineering materials. Generally speaking, degradation of mechanical properties is observed (referred to as thermal softening), accompanied by increasing values of thermal properties. The numerical examples presented below will investigate the qualitative and quantitative influence of thermomechanical coupling in the set of Eqs (12)-(18) and (24). 


\section{RESULTS}

\subsection{Numerical implementation}

The constitutive model is implemented into numerical subroutines by the use of the fully implicit backward Euler scheme (which is always stable and very accurate) and the Newton-Raphson method. The iterative solution procedure is defined as

$\Delta \mathbf{Y}^{(k+1)}=\Delta \mathbf{Y}^{(k)}-\left[\mathbf{J}^{(k)}\right]^{-1} \mathbf{R}^{\operatorname{res}(k)}$

where $\Delta \mathbf{Y}=\left\{\Delta V_{\alpha}\right\}$ is the vector containing the increments of the unknowns, $[\mathbf{J}]=\partial \mathbf{R}^{\text {res }} / \partial \Delta \mathbf{Y}$ is the Jacobian matrix and $\mathbf{R}^{\text {res }}=\left\{R_{\Delta Y_{i}}\right\}$ is a residual vector containing the components $R_{\Delta Y_{i}}=\Delta Y_{i}-\Delta \hat{Y}_{i}$, where $\Delta Y_{i}$ is a variable while $\Delta \hat{Y}_{i}$ denotes the function resulting from the evolution rule for $i$-th variable $Y_{i}$. In the case of uniaxial tension/compression, it is

$\Delta \mathbf{Y}=\left[\begin{array}{c}\Delta \varepsilon \\ \Delta \theta \\ \Delta \lambda \\ \Delta \boldsymbol{\alpha}\end{array}\right], \mathbf{R}^{\text {res }}=\left[\begin{array}{l}\mathbf{R}_{\Delta \varepsilon} \\ R_{\Delta \theta} \\ R_{\Delta \lambda} \\ \mathbf{R}_{\Delta \alpha}\end{array}\right], \mathbf{J}=\left[\begin{array}{cccc}\frac{\partial \mathbf{R}_{\Delta \varepsilon}}{\partial \varepsilon} & \frac{\partial \mathbf{R}_{\Delta \varepsilon}}{\partial \theta} & \frac{\partial \mathbf{R}_{\Delta \varepsilon}}{\partial \alpha} & \frac{\partial \mathbf{R}_{\Delta \varepsilon}}{\partial \lambda} \\ \frac{\partial R_{\Delta \theta}}{\partial \varepsilon} & \frac{\partial R_{\Delta \theta}}{\partial \theta} & \frac{\partial R_{\Delta \theta}}{\partial \alpha} & \frac{\partial R_{\Delta \theta}}{\partial \lambda} \\ \frac{\partial R_{\Delta \lambda}}{\partial \varepsilon} & \frac{\partial R_{\Delta \lambda}}{\partial \theta} & \frac{\partial R_{\Delta \lambda}}{\partial \alpha} & \frac{\partial R_{\Delta \lambda}}{\partial \lambda} \\ \frac{\partial \mathbf{R}_{\Delta \alpha}}{\partial \varepsilon} & \frac{\partial \mathbf{R}_{\Delta \alpha}}{\partial \theta} & \frac{\partial \mathbf{R}_{\Delta \alpha}}{\partial \alpha} & \frac{\partial \mathbf{R}_{\Delta \alpha}}{\partial \lambda}\end{array}\right]$

The condition $\mathbf{R}^{\text {res }}(\Delta \mathbf{Y})=\mathbf{0}$ defines the solution; therefore, the iteration procedure is stopped when the norm of $\mathbf{R}^{\text {res }}$ is sufficiently small.

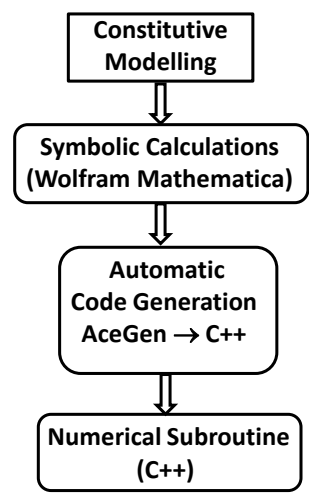

Fig. 3. Scheme of numerical implementation

To solve the problem numerically, the classical concept of elastic predictor/plastic corrector was applied. Wolfram Mathematica 11 (Wolfram Mathematica 11.2, 2017) software was used to generate the expressions for components of the Jacobian matrix. Numerical subroutines were automatically generated in C++ language by the use of AceGen software (Korelc, 2016) (see Fig. 3).

\subsection{Material data}

Experimental observations indicate that P91 steel is not a stable material during fatigue, i.e. the steel microstructure can be modified by the thermomechanical cycle. This is the case, however, only when the temperature reaches or exceeds the tempering temperature during some period of time (Zhang et al., 2002). In general, there are two temperature ranges (separated by tempering temperature) in which the fatigue behaviour of P91 steel is different. Above the tempering temperature, pronounced effects of ageing are observed, additionally enhanced by the fatigue loading. In this temperature range, changes in the mechanical properties of steel depend not only on the current temperature, but also on its history. Identification of model parameters should, therefore, take place jointly for all temperatures in a given load programme (Cailletaud et al., 2000). To do this, experimental thermomechanical fatigue tests should be performed and used to identify the temperature history-dependent parameters.

On the other hand, ageing at temperatures below tempering remains almost constant (which is the case considered here) and mechanical properties depend only on the current temperature, and not on its history. This means that there is no need to include all temperatures together in the identification procedure. Instead of that, it is possible to carry out several isothermal tests at different temperatures, identify material parameters in each test temperature and finally to take into account the influence of temperature on the material parameters by the use of interpolation techniques with polynomial or spline functions. In the present analysis, P91 steel was tested in the temperature range between $20^{\circ} \mathrm{C}$ and $600^{\circ} \mathrm{C}$, while the tempering temperature of this steel is $730^{\circ} \mathrm{C}$ $760^{\circ} \mathrm{C}$.

The identification of model parameters for each isothermal test was performed with the application of the SIMULIA-Isight package (Dassault, 2016). Two key components from the point of view of identification were used: "Data Matching", which offers the ability to calculate different error measures of two or more data sets (e.g. stresses obtained from the experiment and stress calculated numerically) and "Optimisation" component, which allows for various methods of finding the minimum of a multivariable function (see Fig. 4). The following vector of normalised material parameters $\bar{P}_{i} \in\langle-1,1\rangle$ was searched (the temperature dependence of Poisson's ratio $v$ and thermal expansion coefficient $\alpha^{\theta}$ was disregarded):

$\bar{P}_{i}=\frac{2 P_{i}-\left(U_{i}+L_{i}\right)}{U_{i}-L_{i}}, P_{i} \in\left\langle L_{i}, U_{i}\right\rangle,\left\{P_{i}\right\}=\left\{\sigma_{\mathrm{y}}, E, \gamma, C, b, Q, H^{\mathrm{R}}\right\}$

Parameters $P_{i}$ are bounded between their respective lower bounds $L_{i}$ and upper bounds $U_{i}$.

In the present analysis, the following error measure was used:

$F_{\text {obj }}(\overline{\mathbf{P}})=w_{1} \sum_{k=1}^{m}\left|\sigma_{k}(\overline{\mathbf{P}})-\sigma_{k}^{\exp }\right|+$

$+m w_{2} \operatorname{Max}\left|\sigma_{j}(\overline{\mathbf{P}})-\sigma_{j}^{\exp }\right|_{j=1, \ldots, m}$

where $\sigma_{k}^{\exp }$ denote the experimental stress data and $\sigma_{k}(\overline{\mathbf{P}})$ are the stress data calculated numerically by the use of current values of model parameters $\bar{P}_{i}$. The objective function is a weighted sum (weights $w_{1}$ and $w_{2}$ ) of two components: the sum of absolute differences between the experimental and numerical data (stress) and the absolute maximal differences between the experimental data and the numerically simulated data. Such function allows to effectively reduce the maximum error $\left(w_{2} \gg w_{1}\right)$ or to adjust very well the data in the entire tested range $\left(w_{1} \gg w_{2}\right)$.

The search time depends strongly on the selection of the starting point. To shorten the "distance" to the optimal solution, the following procedure was used to select the appropriate starting point as close as possible to the optimal solution:

- Elastic parameters (initial yield stress $\sigma_{\mathrm{y}}$ and elastic modulus $E$ ) were determined manually, taking into account the initial part of the first hysteresis loop. 
- Next, considering the entire first hysteresis loop, the approximate values of the kinematic hardening parameters were determined $(\gamma$ and $C$ ) assuming that the isotropic hardening in the first loop can be disregarded $(b=0, Q=0$ and $H^{\mathrm{R}}=0$ ).

- Then, parameters related to isotropic hardening were searched based on several selected hysteresis loops. As a result, approximate values of all material parameters were determined as the starting point for optimisation.
- Finally, the identification of all material parameters $\left(\sigma_{\mathrm{y}}, E, \gamma, C, b, Q, H^{\mathrm{R}}\right)$ was carried out again, but in a substantially reduced range around the starting point.

The results of the identification procedure described above are presented in Table 1. They ensure the best match of numerical results to experimental results for the selected objective function $F_{\mathrm{obj}}(\overline{\mathbf{P}})$ and the assumed constitutive model.

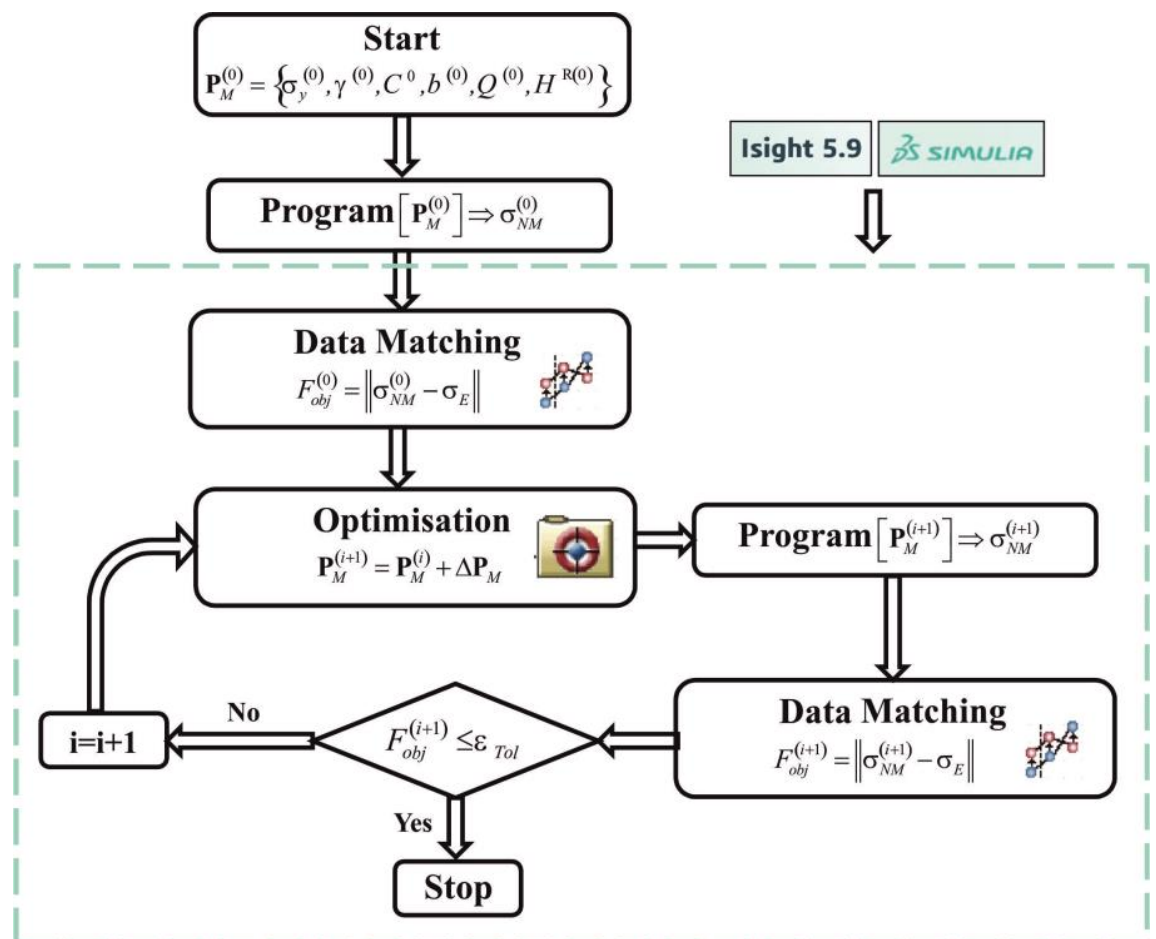

Fig. 4. Algorithm of parameter identification

Table 1. Material parameters $\left(\varepsilon_{\mathrm{ac}}=0.5 \%\right)$

\begin{tabular}{|c|c|c|c|}
\hline & $\mathbf{2 0}{ }^{\circ} \mathrm{C}$ & $\mathbf{4 0 0}{ }^{\circ} \mathrm{C}$ & $\mathbf{6 0 0}{ }^{\circ} \mathbf{C}$ \\
\hline$\sigma_{\mathrm{y}}(\mathrm{MPa})$ & 371 & 281 & 199 \\
\hline$E(\mathrm{MPa})$ & 210464 & 184782 & 173791 \\
\hline$v$ & 0.3 & 0.3 & 0.3 \\
\hline$\gamma$ & 634 & 658.7 & 663.5 \\
\hline$C(\mathrm{MPa})$ & 125806 & 123726 & 99433 \\
\hline$b$ & 0.68 & 1.17 & 3.27 \\
\hline$Q(\mathrm{MPa})$ & -45.5 & -47.7 & -66.3 \\
\hline$H^{\mathrm{R}}(\mathrm{MPa})$ & -1.27 & -2.61 & -5.46 \\
\hline
\end{tabular}

The influence of temperature on the material parameters was then determined by interpolation techniques with spline functions according to the following scheme:

$g(\theta)=a_{i}\left(\theta-\theta_{i}\right)^{3}+b_{i}\left(\theta-\theta_{i}\right)^{2}+c_{i}\left(\theta-\theta_{i}\right)+d_{i}$

for $\theta_{i} \leq \theta \leq \theta_{i+1}$

To illustrate the significance of temperature influence, the temperature-dependent material characteristics are plotted in Fig. 5.

\subsection{Validation}

The general procedure of constitutive modelling, numerical implementation and parameter identification was validated with the use of available experimental data. A very good agreement was obtained between numerical simulations of cyclic softening behaviour and experimental tests (see Fig. 6). 
a) $\quad E(\mathrm{GPa})$

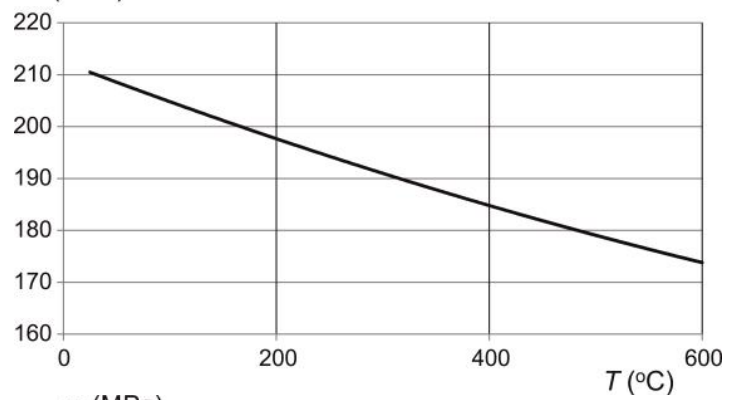

b)

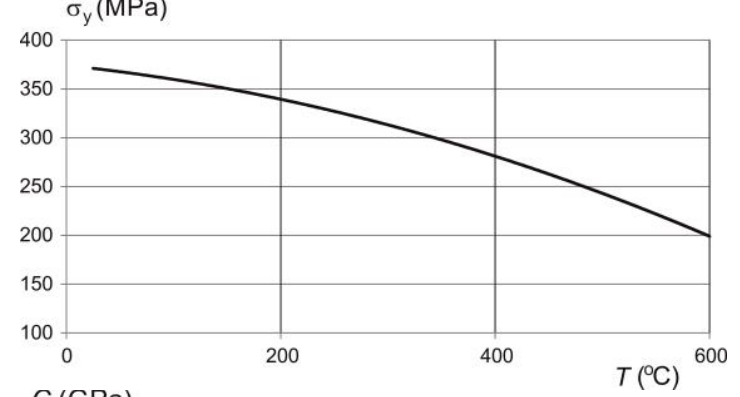

c)

$$
C \text { (GPa) }
$$

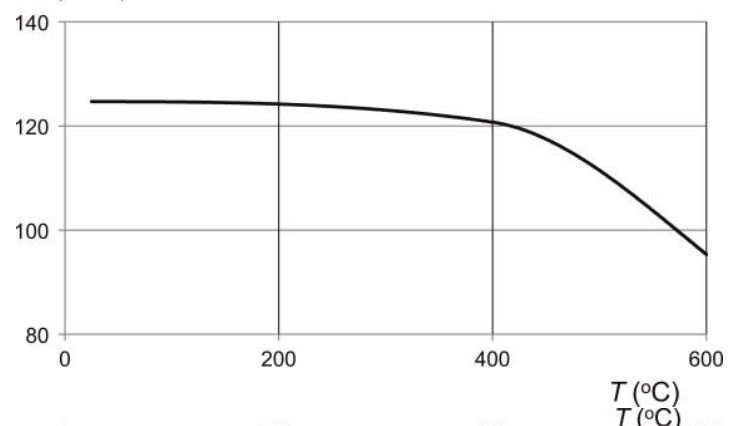

d)

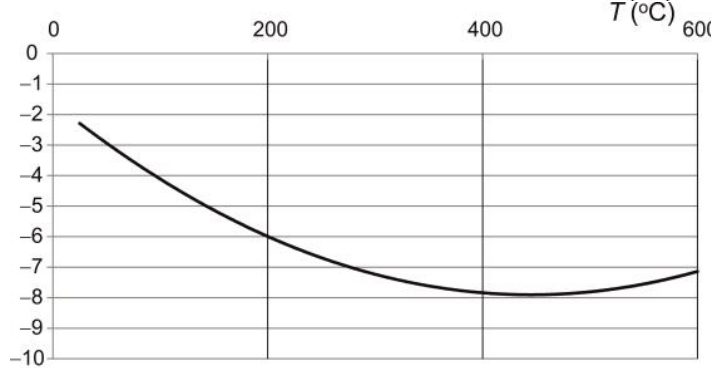

$$
H^{R}(\mathrm{MPa})
$$

e)

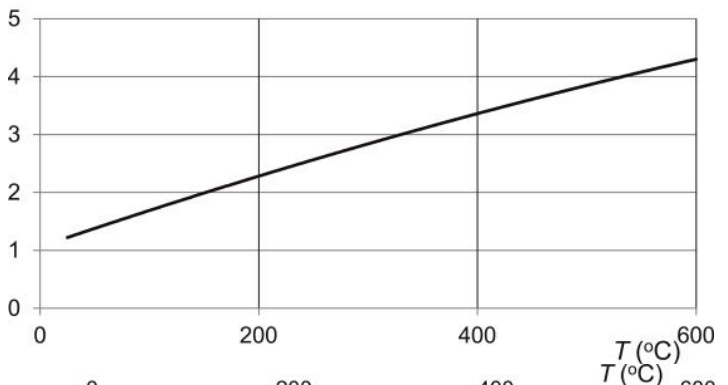

f)

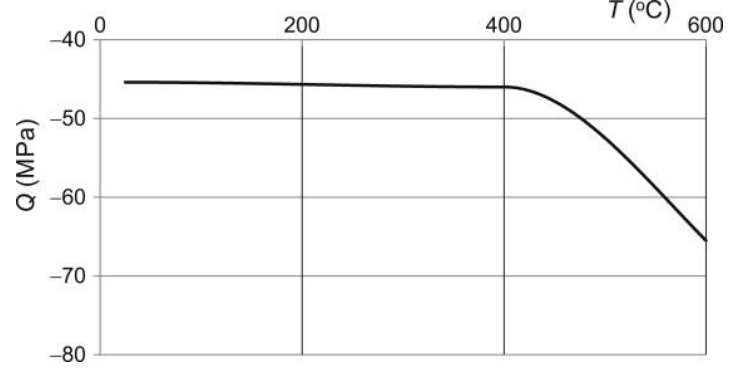

g)

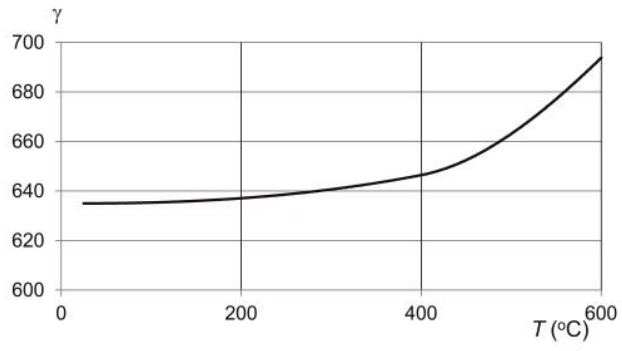

Fig. 5. Function interpolations of material characteristics ( $\mathrm{T}$ denotes temperature expressed in Celsius degrees)

a)

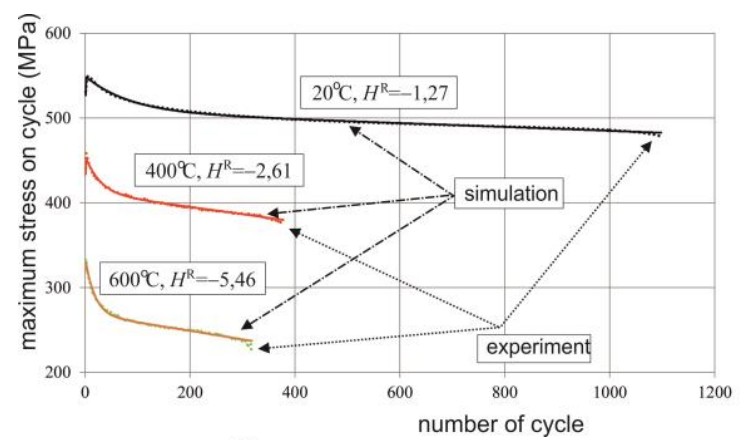

b)

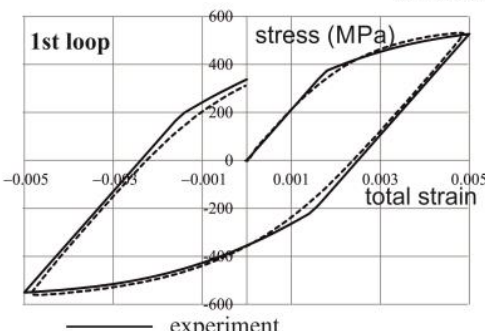

............... simulation

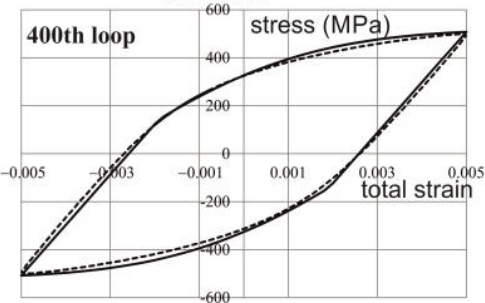

Fig. 6. Validation tests: (a) maximum stress on cycle versus the number of cycles; (b) chosen hysteresis loops at a temperature of $20^{\circ} \mathrm{C}$

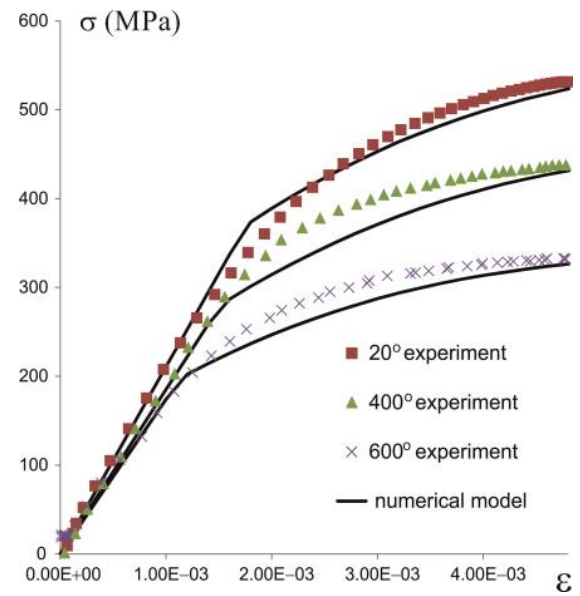

Fig. 7. Influence of linear part of isotropic softening (isothermal fatigue test at a temperature of $20^{\circ} \mathrm{C}$ ) 
The identification procedure allowed to fit very well into the softening curve, while the monotonic stress-strain curve (and individual hysteresis loops) exhibited some differences in the shape between the experiment and numerical simulations (see Fig. 7). This is most likely due to a single back stress used in the presented analysis. Increasing the number of terms in the back stress decomposition would allow the expression of a more extensive strain domain and a better description of the soft transition between elasticity and the onset of plastic flow. On the other hand, it would increase the number of model parameters, and this, in turn, would worsen some other aspects of the model behaviour. The increasing number of model parameters usually leads to multiple local minima of the error function, resulting in a nonunique set of optimal model parameters. Secondly, with a large number of parameters, the model becomes sensitive to their changes, so that a small change in a parameter may cause significant discrepancies in the simulation results.

In the subsequent sections, parametric studies were performed to show the influence of subsequent effects regarded in the constitutive description on the model response.

\subsection{Isothermal tests}

The influence of a linear term in Eq. (6) is shown in Fig. 8. Without the linear term in Eq. (6) $\left(H^{\mathrm{R}}=0\right)$, the shape of the maximum stress evolution cannot be reflected accurately.

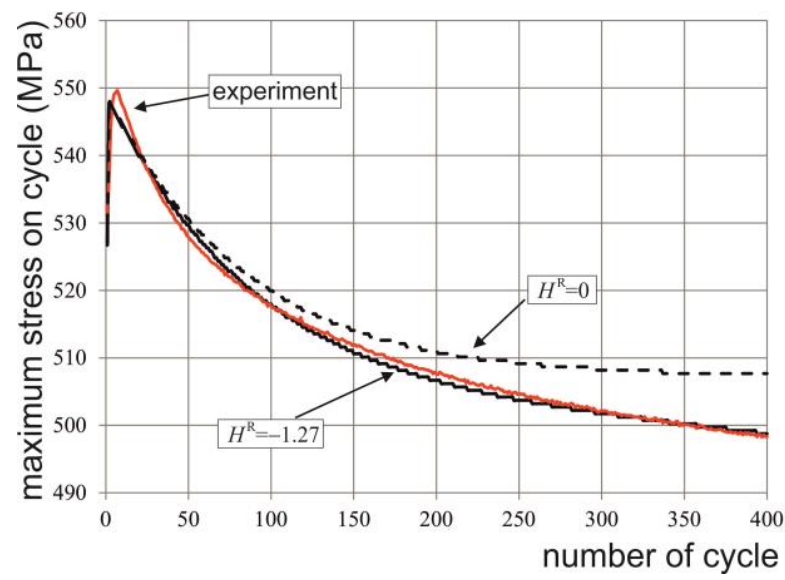

Fig. 8. Influence of linear part of isotropic softening (isothermal fatigue test at a temperature of $20^{\circ} \mathrm{C}$ )

It is possible to fit well into the first stage of cyclic softening, but then the second stage is highly erroneous and unrealistic (the rate of the maximum stress evolution drops to zero, cf. e.g. Saad et al., 2013). If both first and second stages are subjected to parameter optimisation described in the previous section, the error is unacceptably large and the simulated curve substantially differs from the experimental one for both, regarding here stages of material softening. The linear term $R_{2}$ included in Eq. (6) allows obtaining a very good compatibility between experimental and numerical results in the whole softening range considered.

\subsection{Non-isothermal simulations}

Several non-isothermal fatigue tests were subjected to numer- ical simulation according to the strain and temperature control scheme presented in Figs 9 and 10. Reversed strain cycles are considered in Fig. 9, with temperature changes taking place at the maximum and minimum total strain (in-phase and out-of-phase configurations are considered, cf. Fig. 9a,b). During the strain change, the temperature is kept constant. To evaluate the influence of thermomechanical coupling terms in Eqs (12)-(18), the following two cases are compared:

(case 1) Temperature rate terms in kinetic Eqs (12)-(14) are disregarded and the influence of temperature changes is accounted for only by updating the material characteristics;

(case 2) All temperature rate-dependent terms are included (full thermomechanical coupling).

a)
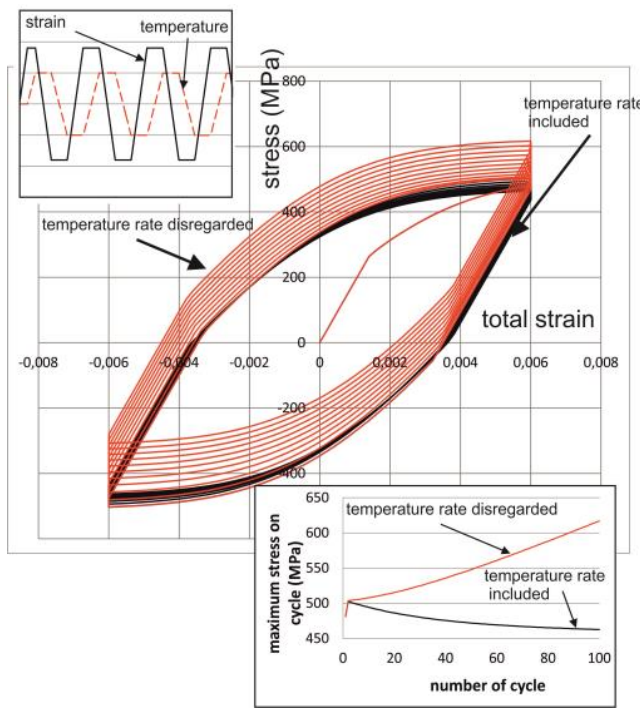

b)

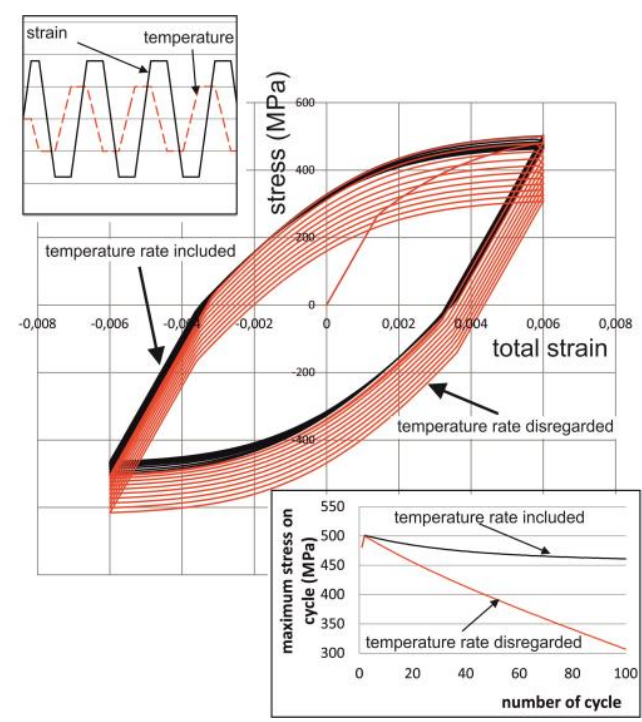

Fig. 9. Non-isothermal fatigue simulations with cyclic temperature change

The stress-strain loops for both cases are presented in Fig. 9a,b. Qualitatively, different results are obtained: without temperature rate terms (case 1), the response exhibits shift of hysteresis loops along the stress axis, while including the temperature rate terms (case 2) allows for preserving stable behaviour. Such an effect was already indicated by Chaboche (2008), and also by Egner and Egner (2016), Egner and Egner (2014) and 
Besson et al. (2009). Due to this unreasonable shift, the simulated maximum stress on cycle contains a substantial error when the temperature rate-dependent terms in Eqs (12)-(14) are disregarded.
The significance of temperature rate influence depends on the specific case of the strain/temperature control scheme. For example, if cyclic strain change is accompanied by monotonic temperature increase or decrease, the difference between case (1) and case (2) is not so pronounced (see Fig. 10).

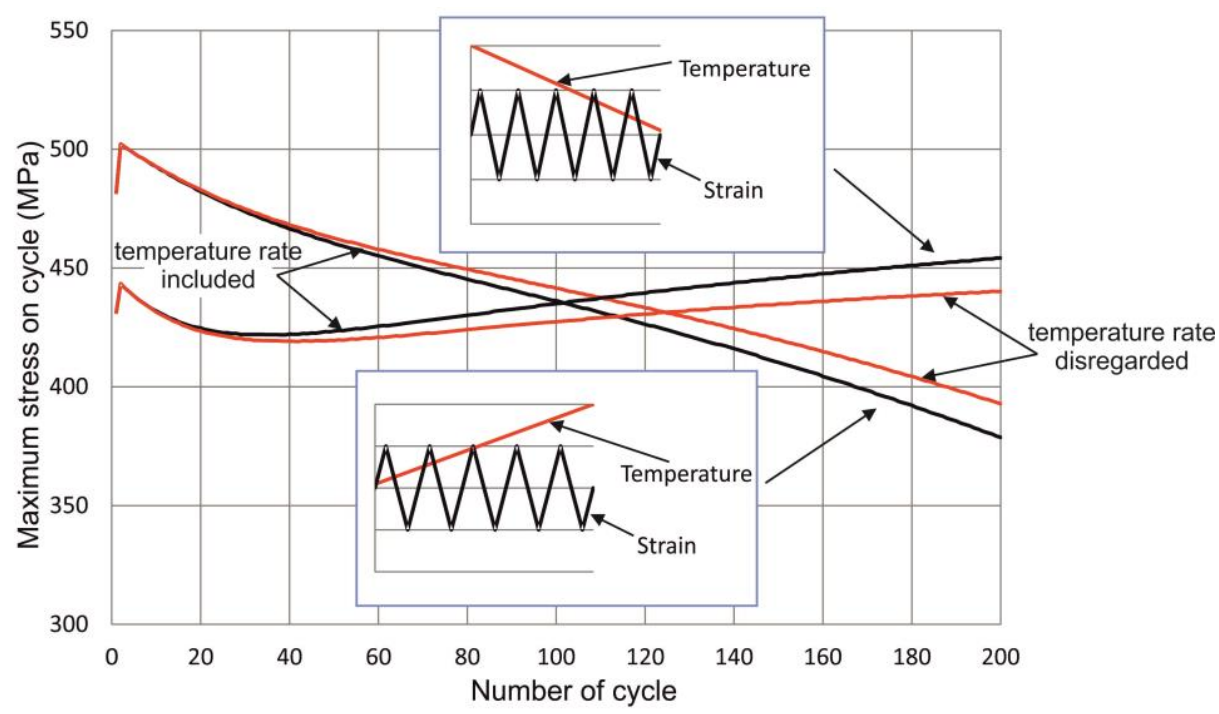

Fig. 10. Non-isothermal fatigue simulations with monotonic temperature change

\section{CONCLUSIONS}

This article is concerned with the description of the cyclic softening of P91 steel under non-isothermal conditions. Particular attention is paid to accounting for thermomechanical coupling in constitutive modelling and to examining the effect of this coupling on the model's response. The analysis presented in this work consists of six stages: (1) experimental testing of the material at several test temperatures, (2) constitutive modelling concerning variable temperature influence and the phenomenon of material cyclic softening, (3) numerical implementation of the mathematical model, (4) identification of model parameters at different test temperatures and then interpolating the results to obtain temperature-dependent material characteristics, (5) validating the analysis by comparing the experimental and numerical results and (6) investigating the effect of thermomechanical coupling on the model response, depending on the thermomechanical loading scheme.

To investigate the effect of full thermomechanical coupling on the model response, the classical constitutive model of Armstrong and Frederick (Frederick and Armstrong, 2007) was extended to take account of the rate of temperature explicitly. To properly reflect the first two stages of material cyclic softening, in the description of the isotropic plastic hardening, the drag stress was decomposed into two components - one of them responsible for the first stage of rapid softening, while the other for the quasilinear softening stage. The influence of temperature on material parameters was determined using interpolation techniques with polynomial spline functions. The numerical test results revealed a significant qualitative and quantitative effect of temperature. This means that the coupling between temperature and dissipative phenomena occurring in the material can have a significant impact on the response of the constitutive model. Disregarding the temperature rate in the model equations can, therefore, lead to erro- neous results, while the amount of error depends on the thermomechanical cyclic load configuration.

The constitutive description presented in this work was based on a model of the Armstrong and Frederick type. Such a model is not capable to reflect all the physical mechanisms able to produce material nonlinearities on a macro scale (such as unilateral damage, plastic strain range memorisation effect, etc.). Nevertheless, even this relatively simple model involves seven parameters that have to be identified for several temperatures of fatigue tests. This is a laborious and time-consuming task, and each additional parameter significantly increases the size of the problem. Therefore, in the presented analysis, a relatively simple model is considered, but the described procedure is general and can also be applied to more comprehensive modelling (Saanouni and Devalan, 2012).

\section{REFERENCES}

1. Besson J., Cailletaud G., Chaboche S. (2009), Non-linear mechanics of materials, Springer.

2. Cailletaud G., Depoid C., Massinon D., Nicouleau-Bourles E. (2000), Elastoviscoplasticity with ageing in aluminium alloys, [In:] Continuum Thermomechanics: The Art and Science of Modelling Material Behaviour (Paul Germain's Anniversary Volume), Solid Mechanics and Its Applications, Kluwer Academic Publishers, 75-86.

3. Cailletaud G., Saï K. (1995), Study of plastic/viscoplastic models with various inelastic mechanisms, International Journal of Plasticity, 11, 991-1005.

4. Chaboche J.L. (2008), A review of some plasticity and viscoplasticity constitutive theories, International Journal of Plasticity, 24(10), 1642-1693.

5. Dassault. (2016), SIMULIA Abaqus Extended Products, Abaqus 6.14 - AP Isight 5.9. http://www.3ds.com/products/simulia

6. Duda P. (2015), Solution of an inverse axisymmetric heat conduction problem in complicated geometry. International Journal of Heat and Mass Transfer, 419-428. 
7. Egner H. (2012), On the full coupling between thermo-plasticity and thermo-damage in thermodynamic modeling of dissipative materials, International Journal of Solids and Structures, 49(2), 279-288.

8. Egner H., Egner W. (2014), Modeling of a tempered martensitic hot work tool steel behavior in the presence of thermo-viscoplastic coupling, International Journal of Plasticity, 57, 77-91.

9. Egner H., Ryś M. (2017), Total energy equivalence in constitutive modeling of multidissipative materials, International Journal of Damage Mechanics, 26(3),, 417-446.

10. Egner W., Egner H. (2016), Thermo-mechanical coupling in constitutive modeling of dissipative materials, International Journal of Solids and Structures, 91, 78-88.

11. Farragher T.F. (2014), Thermomechanical Analysis of P91 Power Plant Components, PhD Thesis, National University of Ireland Galway, https://aran.library.nuigalway.ie/xmlui/handle/10379/4161.

12. Fournier B., Salvi M., Dalle F., De Carlan Y., Caës C., Sauzay M., Pineau A. (2010), Lifetime prediction of $9-12 \% \mathrm{Cr}$ martensitic steels subjected to creep-fatigue at high temperature, International Journal of Fatigue, 32(6), 971-978.

13. Fournier B., Sauzay M., Caës C., Noblecourt M., Mottot M., Bougault A., Rabeau V., Man J., Gillia O., Lemoine P., Pineau A. (2008), Creep-fatigue-oxidation interactions in a $9 \mathrm{Cr}$-1Mo martensitic steel. Part III: Lifetime prediction, International Journal of Fatigue, 30(10-11), 1797-1812.

14. Frederick C.O., Armstrong P.J. (2007), A mathematical representation of the multiaxial Bauschinger effect, Materials at High Temperatures, 24(1), 1-26.

15. Jones W.B., Van Den Avyle J.A. (1980), Substructure and strengthening mechanisms in $2.25 \mathrm{Cr}-1$ Mo steel at elevated temperatures, Metallurgical Transactions, A 11A, 1275-1286.

16. Kannan R., Sankar V., Sandhya R., Mathew M.D. (2013), Comparative evaluation of the low cycle fatigue behaviours of P91 and P92 steels, Procedia Engineering, 55, 149-153

17. Kim S., Weertman J.R. (1988), Investigation of microstructural changes in a ferritic steel caused by high temperature fatigue, Metallurgical Transactions, A 19A, 999-1007.

18. Kruml T., Polák J. (2001), Fatigue softening of X10CrAl24 ferritic steel, Materials Science and Engineering, A 319, 564-568.

19. Kyaw S.T., Rouse J.P., Lu J., Sun W. (2016), Determination of material parameters for a unified viscoplasticity-damage model for a P91 power plant steel, International Journal of Mechanical Sciences, 115-116, 168-179.

20. Li M., Barrett R.A., Scully S., Harrison N.M., Leen S.B., O'Donoghue P.E. (2016), Cyclic plasticity of welded P91 material for simple and complex power plant connections, International Journal of Fatigue, 87, 391-404.

21. Lu J., Sun W., Becker A., Saad A.A. (2015), Simulation of the fatigue behaviour of a power plant steel with a damage variable, International Journal of Mechanical Sciences, 100, 145-157.

22. Mroziński S. (2011), The influence of loading program on the course of fatigue damage cumulation, Journal of Theoretical and Applied Mechanics, 49(1), 83-95.

23. Mroziński S., Golański G. (2014), Influence of temperature change on fatigue properties of P91 steel, Materials Research Innovations, 18 (2), 504-508.

24. Nagesha A., Valsan M., Kannan R., Bhanu Sankara Rao K., Mannan S.L. (2002), Influence of temperature on the low cycle fatigue behaviour of a modified $9 \mathrm{Cr}$-1Mo ferritic steel, International Journal of Fatigue, 1285-1293.

25. Korelc J. (2016), AceGen 6.824 Windows.

26. Saad A.A., Hyde T.H., Sun W., Hyde C.J., Tanner D.W.J. (2013), Characterization of viscoplasticity behaviour of P91 and P92 power plant steels, International Journal of Pressure Vessels and Piping, 111-112, 246-252

27. Saad A.A., Sun W., Hyde T.H., Tanner D.W.J. (2011), Cyclic softening behaviour of a P91 steel under low cycle fatigue at high temperature, Procedia Engineering, 1103-1108.
28. Saanouni K., Devalan P. (2012), Damage Mechanics in Metal Forming: Advanced Modeling and Numerical Simulation, Wiley.

29. Sauzay M., Brillet H., Monnet I., Mottot M., Barcelo F., Fournier B., Pineau A. (2005), Cyclically induced softening due to low-angle boundary annihilation in a martensitic steel, Materials Science and Engineering A, 400-401(1-2), 241-244.

30. Shankar V., Valsan M., Rao K.B.S., Kannan R., Mannan S.L. Pathak S.D. (2006), Low cycle fatigue behavior and microstructural evolution of modified $9 \mathrm{Cr}$-1Mo ferritic steel, Materials Science and Engineering A, 437, 413-422.

31. Skrzypek J.J., Kuna-Ciskał H. (2003), Anisotropic Elastic-BrittleDamage and Fracture Models Based on Irreversible Thermodynamics, Lecture Notes in Applied and Computational Mechanics, 9, 143-184.

32. Sulich P., Egner W., Mroziński S., Egner H. (2017), Modeling of cyclic thermo-elastic-plastic behaviour of P91 steel, Journal of Theoretical and Applied Mechanics, 55(2), 595-606.

33. Taleb L., Cailletaud G. (2010), An updated version of the multimechanism model for cyclic plasticity, International Journal of Plasticity, 26(6), 859-874.

34. Wolfram Mathematica 11.2. (2017), http://www.wolfram.com/mathematica/new-in-11/

35. Xie X., Jiang W., Chen J., Zhang X., Tu S.-T. (2019), Cyclic hardening/softening behavior of $316 \mathrm{~L}$ stainless steel at elevated temperature including strain-rate and strain-range dependence: Experimental and damage-coupled constitutive modeling, International Journal of Plasticity, 114, 196-214.

36. Zhang Z., Delagnes D., Bernhart G. (2002), Anisothermal cyclic plasticity modelling of martensitic steels, International Journal of Fatigue, 24(6), 635-648.

37. Zhao P., Xuan F. Z., Wu D.L. (2017), Cyclic softening behaviors of modified $9-12 \% \mathrm{Cr}$ steel under different loading modes: Role of loading levels, International Journal of Mechanical Sciences, 131-132, 278-285.

38. Zhou J., Sun Z., Kanouté P., Retraint, D. (2018), Experimental analysis and constitutive modelling of cyclic behaviour of $316 \mathrm{~L}$ steels including hardening/softening and strain range memory effect in LCF regime, International Journal of Plasticity, 107, 54-78.

Acknowledgements: This work was supported by the National Science Centre of Poland through the Grant No. 2017/25/B/ST8/02256. 